Historic, Archive Document

Do not assume content reflects current scientific knowledge, policies, or practices. 



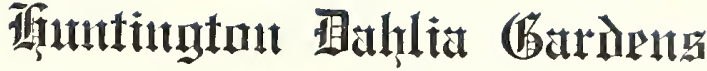

HUNTINGTON, N. Y.

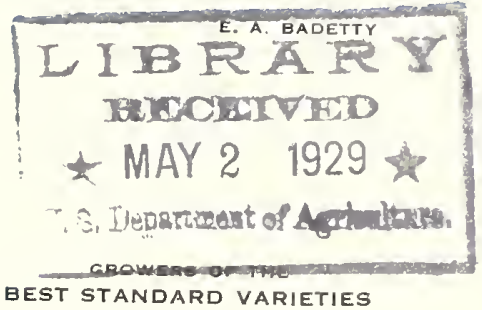

BEST STANDARD VARIETIES

ORIGINATORS OF CHOICE NEW VARIETIES

\section{SPECIAL WHOLESALE PRICES FOR 1929}

All stock is guaranteed to be first grade selected tubers and in all ways up to our high standard of quality. As there is a general shortage of good stock this season, we advise placing your order early while we still have the varieties you want.

Stock will be reserved and shipment made at any future date if a deposit of $25 \%$ is sent with your order. Special quotations given on large orders.

Terms CASH, F. O. B. Huntington, N. Y. All orders will be sent by express collect unless postage is sent. No orders accepted for less than 5 tubers of a variety where the price is less than 50 cents each and no orders will be shipped for less than $\$ 5.00$.

All claims for adjustment must be made immediately upon receipt of tubers. Prices on this list are net and subject to change without notice and cancel all prior quotations. Orders accepted subject to stock on hand.

Ambassador, $\mathrm{C}$. Yellow per division

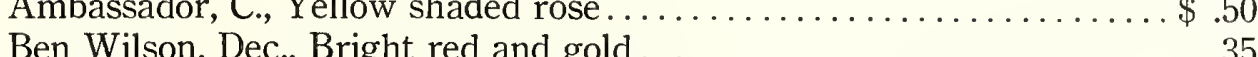

Champagne, Dec., Copper and chamois .................... .60

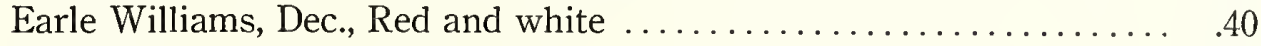

El Dorado, Dec., Pure gold . . . . . . . . . . . . . . . . . . . . . . . . . . . .75

Elite Glory, Dec., Bright red . . . . . . . . . . . . . . . . . . . . 2.00

Ellinor Vandeveer, Dec., Rose pink . . . . . . . . . . . . . . . . . . . $\quad .35$

Faith Gariboldi, Dec., Silvery pink ....................... 1.00

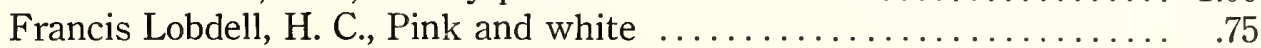

Gladys Sherwood, H. C., Pure white ....................... 25

Hubert Badetty, H. C., Salmon and buff .................... 1.00

Hunter's Moon, H. C., Sulphur yellow . . . . . . . . . . . . . . . .50

Jersey's Beacon, Dec., Chinese scarlet . . . . . . . . . . . . . 1.00

Jersey's Beauty, Dec., Clear pink ....................... ${ }_{.25}$

Kirby's Attraction, H. C., Lavender ..................... 1.00

Lady Helen, H. C., Pink streaked white .................. . 25

Lillian Baldwin, Dec., Deep rose pink ....................... 1.25

Margaret Woodrow Wilson, Dec., Opalescent pink . . . . . . . . . . . 1.50

Marmion, Dec., Large golden yellow .................... 1.50

Mars, H. C., Rich orange . . . . . . . . . . . . . . . . . . . . . $\quad .75$

Mary Bond, Dec., Blended cream and pale rose $(\mathrm{New})$. . . . . . . . . . . 5.00

Merveilleux, Dec., Very large rose pink ................. 1.25

Miss Huntington, Dec., Vivid scarlet (New) . . . . . . . . . . . . 5.00

Miss New York, H. C., Large rich rose ................... 1.25

Mrs. I. de ver Warner, Dec., Mauve pink . . . . . . . . . . . . . . . . . $\quad .10$

Mrs. John Sheepers, Dec., Large yellow and rose . . . . . . . . . . . 35

Pink Triumphant, H. C., Pink ...................... $\quad .50$

Premier George Clemenceau, C., Fancy yellow and white.......... $\quad .75$

Prince of India, Dec., Dark carmine . . . . . . . . . . . . . . . . 2.50

Red Wing, Dec., Bright scarlet....................... $\quad 20$

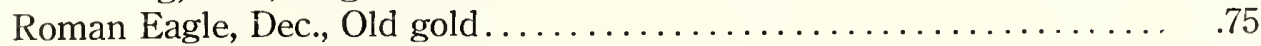

Rosa Nell, Dec., Clear rich rose ....................... $\quad .20$

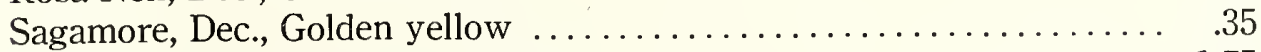

Salbach's White, Dec., Pure white ...................... 1.75

Seedling No. 508, Dec., Deep pink ........................... 1.00

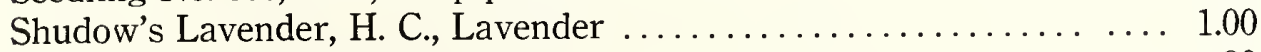

Snowdrift, Dec., Large pure white $\ldots \ldots \ldots \ldots \ldots \ldots \ldots \ldots \ldots \ldots \ldots \ldots . . . \ldots \ldots$

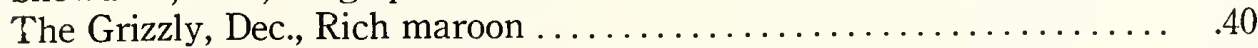

The U. S. A., H. C., Orange ....................... .40

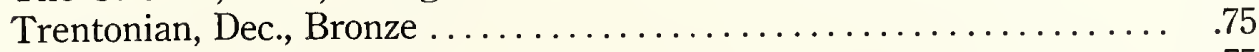

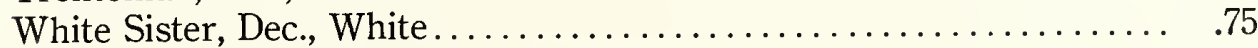

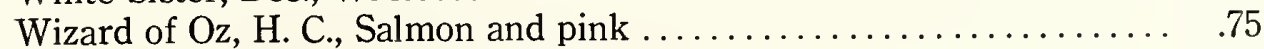

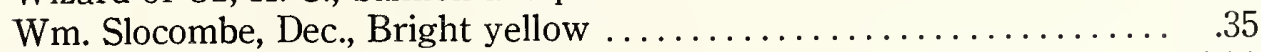

Yellow Bird, Dec., Rich deep yellow ........................ 2.50

Yellow de Luxe, Dec., Buttercup yellow ................... 2.00 
